\title{
Supporting Information for Propagating Hybrid Tamm Exciton Polaritons in Organic Microcavity
}

\author{
Bin $\mathrm{Liu}^{1}$, Rong $\mathrm{Wu}^{1,2}$, Vinod M. Menon ${ }^{1,2^{*}}$ \\ ${ }^{1}$ Department of Physics, City College of New York, New York, NY 10031, USA \\ ${ }^{2}$ Department of Physics, Graduate Center of the City University of New York (CUNY), \\ New York, NY 10016, USA \\ * Email: vmenon@ccny.cuny.edu
}




\section{Optical Spectroscopy Characterization Experiment}

Figure S1 shows the schematic of optical spectroscopy characterization setup. ${ }^{1}$ For angle-resolved reflectivity measurements, as shown in Figure S1(a), a broadband of white light from tungsten halogen lamp was focused onto the sample by a 50x objective with a high numerical aperture $(\mathrm{NA}=0.8)$, and the reflected signal was collected by the same 50x objective, covering an angular range of $\pm 53.1^{\circ}$. Using a converging lens with long focal length, the angle-resolved reflectivity was measured by the deflection using the spectrometer (Princeton Instruments, Acton SpectraPro SP2500) and charge-coupled device (CCD) camera (Princeton Instruments, PIX 1024B). The polarization is determined by a polarizer $(\mathrm{P})$.

For angle-resolved PL measurements, a fiber-coupled CW blue diode at $462 \mathrm{~nm}$ with a laser line filter was used to excite the sample, and the pump beam was focused onto the sample using the $50 \mathrm{x}$ objective $(\mathrm{NA}=0.8)$ with a spot size of $5 \mu \mathrm{m}$ in diameter, and the emission signal was collected by the same 50x objective. A $500 \mathrm{~nm}$ longpass filter (LP) was used to block the residual excitation beam, shown in Figure S1(a), and a combination of half-wave plate (HWP) and polarizer (P1) was used to determine the polarization of incident beam and continuously vary the pump power, which gave those polarization and pump-power dependent measurement results.

To investigate the propagation property, as shown in Figure S1(b), the converging lens f1 was replaced by lens f2 with a short focal length so that the real plane locates onto the slit of the spectrometer. The space image of the wavelength-resolved emission from the sample was recorded using the CCD camera. ${ }^{2}$

\section{Electric-field-intensity Distribution Profile Simulation}

To better understand the experimental observation of reflectivity from different cavities, the wavelength-resolved electric-field-intensity distribution is simulated using transfer matrix method. 
Figure S13 shows the simulated profile of electric-field-intensity distribution for both metal cavity and hybrid cavity filled with a $115 \mathrm{~nm}$ non-absorbing polystyrene layer at normal incidence. For the metal cavity, as shown in Figure S13(a), the electric field is enhanced in the cavity mode of $530 \mathrm{~nm}$. For $\mathrm{HC} 1$, the electric field of the cavity mode $(\sim 530 \mathrm{~nm})$ is locally enhanced within the cavity, while the electric field is locally enhanced at the interface between the intermediate Ag layer and DBR for the TP mode of 450nm (Figure S13(b)), indicating very weak interaction. After red-shifting TP mode by inserting a $30 \mathrm{~nm} \mathrm{Si}_{3} \mathrm{~N}_{4}$ layer between $\mathrm{Ag}$ and DBR (HC2), the electric field enhancement of two modes $(\sim 470$ and $540 \mathrm{~nm})$ are evidently noted both within the metal cavity and at the $\mathrm{Ag} / \mathrm{Si}_{3} \mathrm{~N}_{4}$ interface (Figure S13(c)), which attributes to the strong coupling between cavity mode and TP. Figure S13(d) illustrates the corresponding profile of the electricfield-intensity distribution for a hybrid cavity with a $50 \mathrm{~nm} \mathrm{Si}{ }_{3} \mathrm{~N}_{4}$ layer (HC3), again, two modes ( 500 and $550 \mathrm{~nm}$ ) have enhanced electric field both within the metal cavity and at the $\mathrm{Ag} / \mathrm{Si}_{3} \mathrm{~N}_{4}$ interface. Compared to the results from $\mathrm{HC} 2$, each mode of $\mathrm{HC} 3$ has closer electric field intensity within the metal cavity and at the $\mathrm{Ag} / \mathrm{Si}_{3} \mathrm{~N}_{4}$ interface, which means the TP mode is more resonant with the cavity mode in $\mathrm{HC} 3$ at the particular angle (normal incidence) studied.

Figure S14 shows the simulated profile of electric-field-intensity distribution for both metal cavity and hybrid cavity filled with a $115 \mathrm{~nm}$ BODIPY/PS layer at normal incidence. For the metal cavity, as shown in Figure S14(a), the electric field is enhanced in both upper and lower polariton modes $(\sim 500$ and $560 \mathrm{~nm})$. For HC1, the electric field of the cavity polariton modes $(\sim 500$ and 560 $\mathrm{nm}$ ) is locally enhanced within the metal cavity, while the electric field is locally enhanced at the interface between the intermediate Ag layer and DBR for the TP mode of 450nm (Figure S14(b)). Compared to stand-alone metal cavity, there is no energetic variation of cavity polariton modes in $\mathrm{HC1}$, and TP mode is also unchanged comparing with that calculated in the PS-filled HC1, 
therefore, no strong coupling occurs between cavity polariton and TP. Figure S14(c) shows the electric field distribution for $\mathrm{HC} 2$ with a $30 \mathrm{~nm} \mathrm{Si} \mathrm{N}_{4}$ layer between $\mathrm{Ag}$ and DBR, the field enhancement of three modes $(\sim 475,516$ and $555 \mathrm{~nm})$ are evidently noted, which correspond to upper, middle, and lower hybrid polariton, resulting from the strong coupling between cavity polariton and TP. Figure S14(d) illustrates the electric field distribution for a hybrid cavity with a $50 \mathrm{~nm} \mathrm{Si}_{3} \mathrm{~N}_{4}$ layer (HC3), and two modes ( 485 and $555 \mathrm{~nm}$ ) have significantly enhanced electric field, which correspond to upper and lower hybrid polariton. The field enhancement of the middle hybrid polariton is less visible at normal incidence, which agrees with the experimental observation in Figure 2(g) in the main paper.

\section{Exponential Fitting for the Real-space PL Intensity Profile}

The wavelength-resolved real-space PL intensity data from all the samples are fit using exponential decay functions. It has to be mentioned that no good fitting is obtained when the fitting is taken starting from the PL peak no matter how many exponentials are used, and we believe this due to the dramatically different decay behaviors at the very beginning of the propagation, where the PL peak is from the local illuminated area determined by the pump beam spot. As the propagation property is principally dominated by the long decay tail of the PL intensity profile, it is reasonably practicable to do a tail fit starting from the PL intensity of around $40 \%$ of the PL peak. It is worth noting that, as the $\mathrm{MC}$ and film sample have no multiple pairs of $\mathrm{SiO}_{2}$ and $\mathrm{Si}_{3} \mathrm{~N}_{4}$, they show different experimental baseline background (Figure S15 and S16) for PL compared to all the HC samples, however, this does not affect the fitting results for the propagation length. We have also tried to do the tail fit starting from the PL intensity of 50\% and 30\% of the PL peak, and the fitting results are the same, including the offset of the baseline background. 
One-exponential function can well fit the PL intensity data from the BODIPY/PS neat film spin-cast on $30 \mathrm{~nm} \mathrm{Ag} \mathrm{coated} \mathrm{glass,} \mathrm{as} \mathrm{shown} \mathrm{in} \mathrm{Figure} \mathrm{S16,} \mathrm{and} \mathrm{the} \mathrm{fitting} \mathrm{constant}(\sim 0.66 \mu \mathrm{m})$ is almost the same for those PL profiles at different wavelengths studied. For all the cavity samples, bi-exponential function is used to fit the PL intensity data, as listed in Table S2, the first exponential fitting constant from all the cavities is always smaller than $0.8 \mu \mathrm{m}$, which is close to the fitting constant $(\sim 0.66 \mu \mathrm{m})$ from the neat film, while the second exponential fitting constant with larger values vary with different cavities as discussed in the main paper. As the second exponential can trace the longer tail of PL intensity profile from those cavity samples, the second fitting decay constant (larger) can be used to interpret the propagation length of cavity polariton from metal cavity (MC) and hybrid polariton from hybrid cavity (HC). It should be mentioned that bi-exponential function is tried to fit the $540 \mathrm{~nm}$ PL data from MC, yielding two equal fitting constants, thus, one-exponential function instead is used, which gives the same fitting constant of $\sim 1.63 \mu \mathrm{m}$ given in Table $\mathbf{S 2}$. 


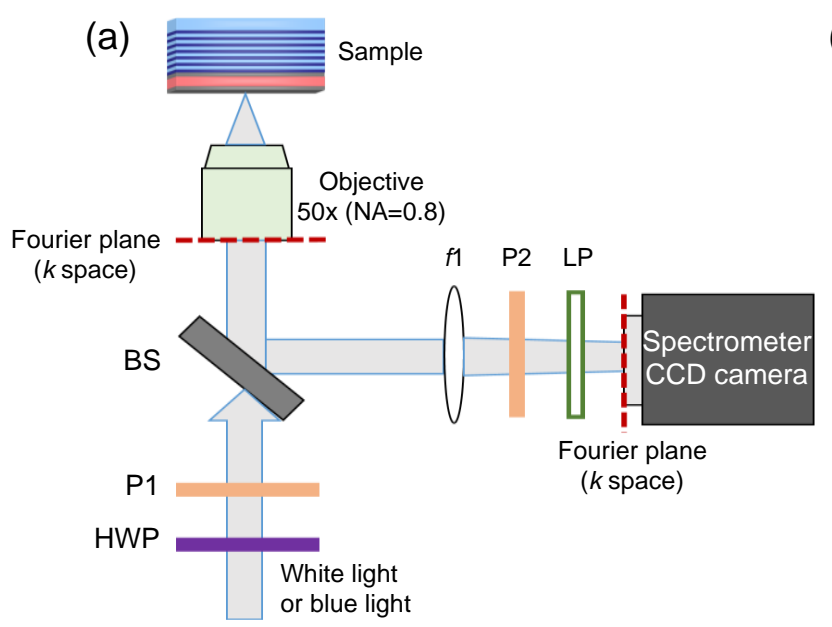

(b)

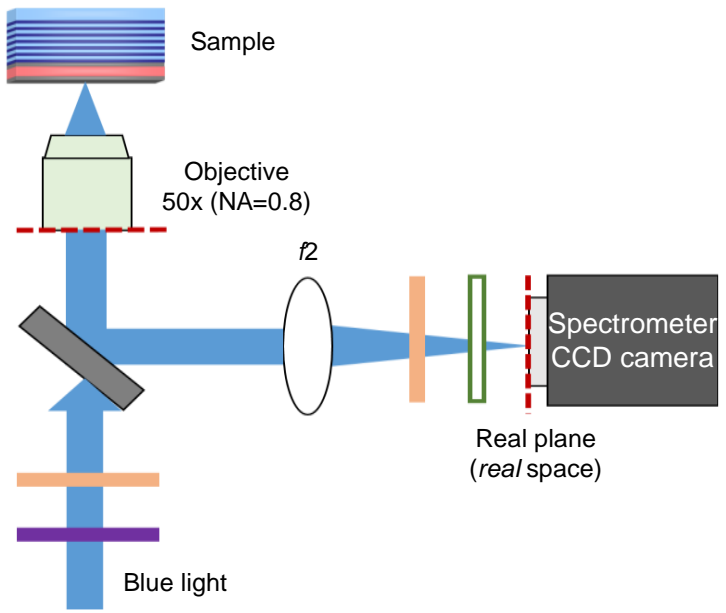

Figure S1. Schematic of optical spectroscopy characterization setup. a) Angle-resolved reflectivity/photoluminescence in $k$ space. b) Wavelength-resolved PL in real space. BS: beam splitter; HWP: half-wave plate; P1,2: polarizer; f1,2: converging lens; LP: $500 \mathrm{~nm}$ longpass filter. 

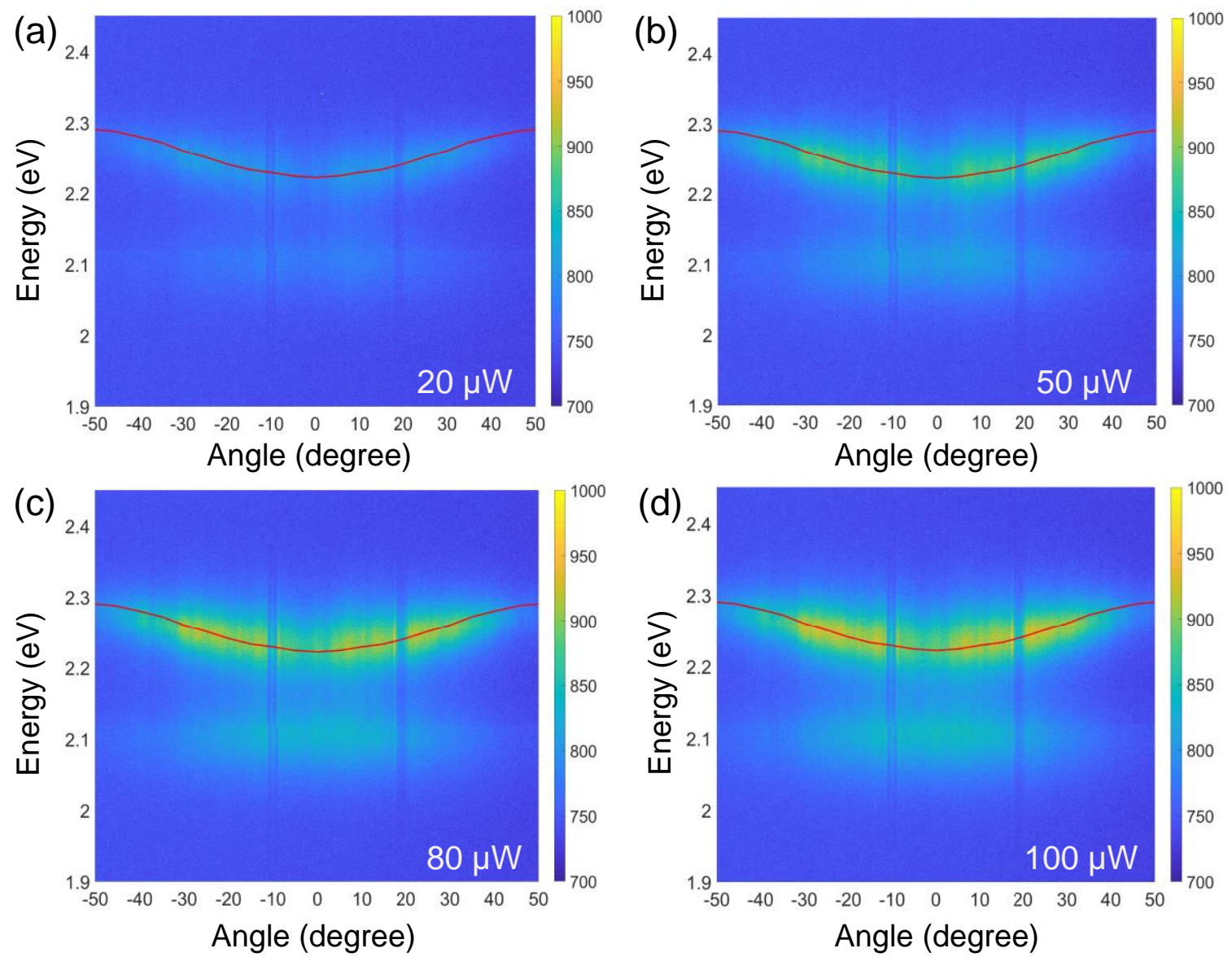

Figure S2. TE-polarized angle-resolved photoluminescence map measured for the metal cavity (MC) under different pump power: a) $20 \mu \mathrm{W}$, b) $50 \mu \mathrm{W}$, c) $80 \mu \mathrm{W}$, d) $100 \mu \mathrm{W}$. The red curve indicates the lower polariton dispersion. 

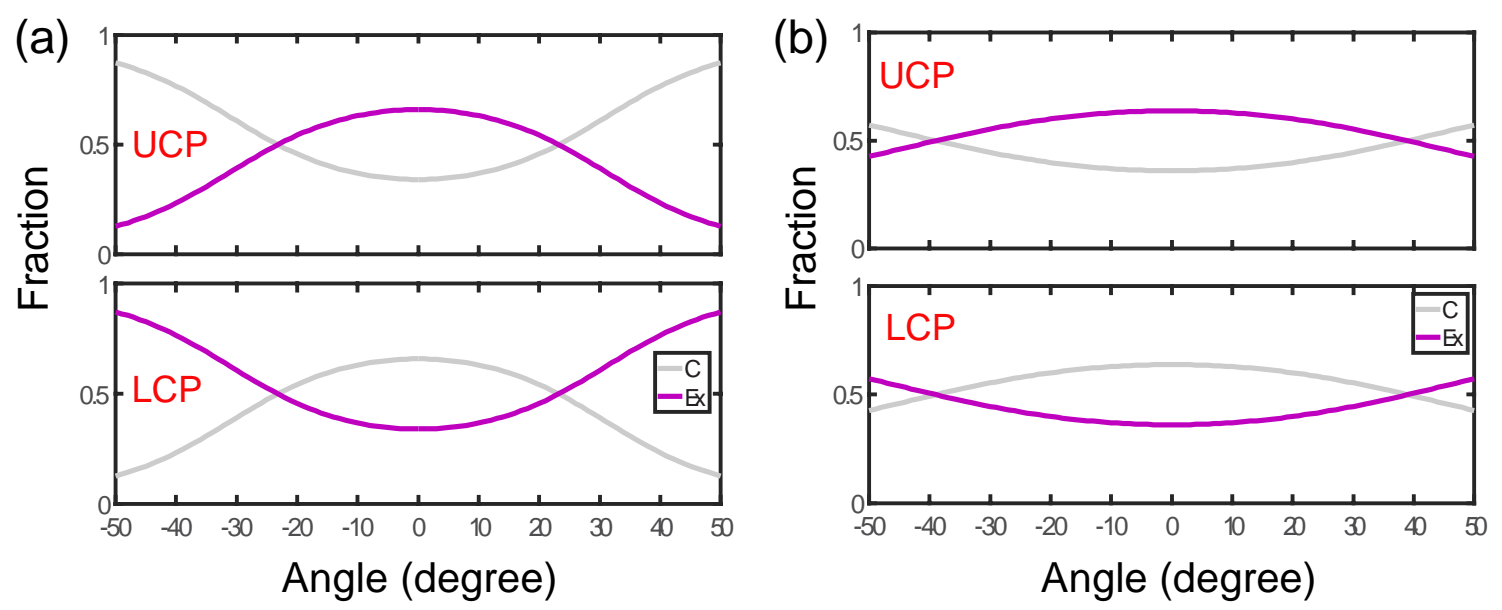

Figure S3. Hopfield coefficients $|\alpha|^{2}$ and $|\beta|^{2}$ showing the composition of UCP and LCP calculated using the coupled two-oscillator model for the metal cavity. a) TE polarization, b) TM polarization. C: cavity photon, Ex: exciton. 

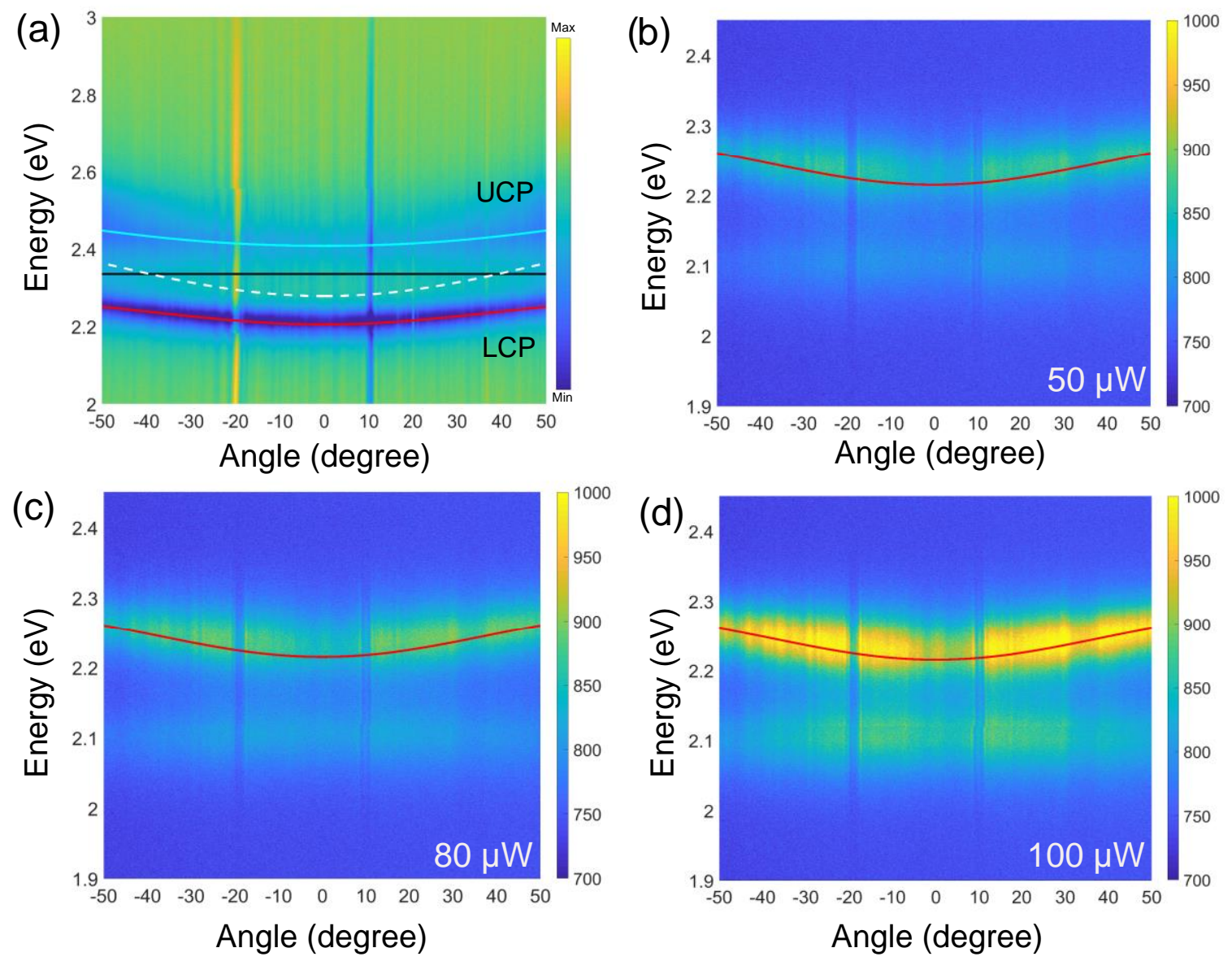

Figure S4. a) TM-polarized angle-resolved reflectivity map measured for the metal cavity. The solid black and dashed white curve show the uncoupled exciton and cavity photon dispersion. The solid cyan and red curve show the cavity polariton dispersion. Angle-resolved photoluminescence map: b) $50 \mu \mathrm{W}$, c) $80 \mu \mathrm{W}$, d) $100 \mu \mathrm{W}$. 

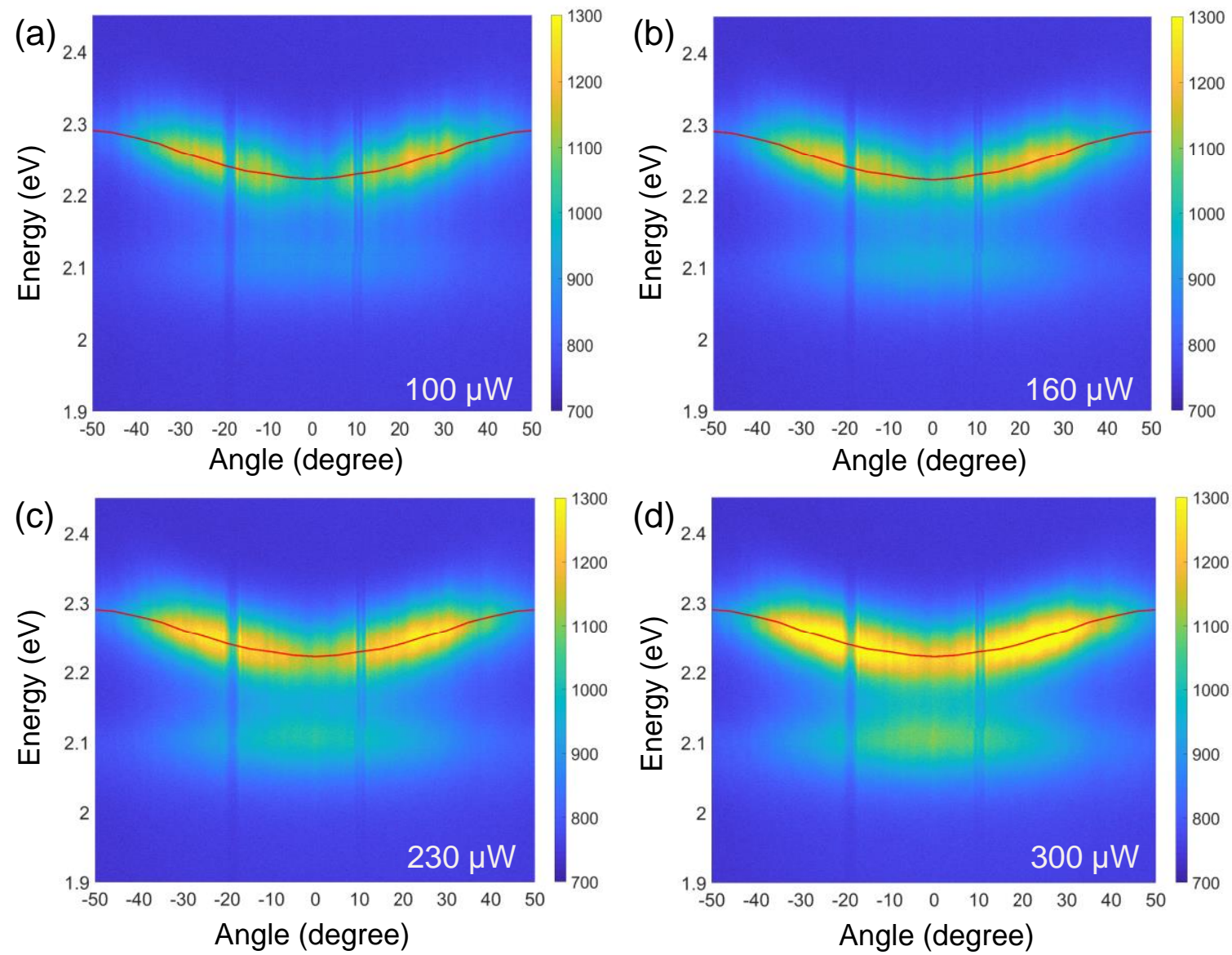

Figure S5. TE-polarized angle-resolved photoluminescence map measured for the hybrid cavity with no top $\mathrm{Si}_{3} \mathrm{~N}_{4}$ layer (HC1) under different pump power: a) $100 \mu \mathrm{W}$, b) $160 \mu \mathrm{W}$, c) $230 \mu \mathrm{W}$, d) $300 \mu \mathrm{W}$. 

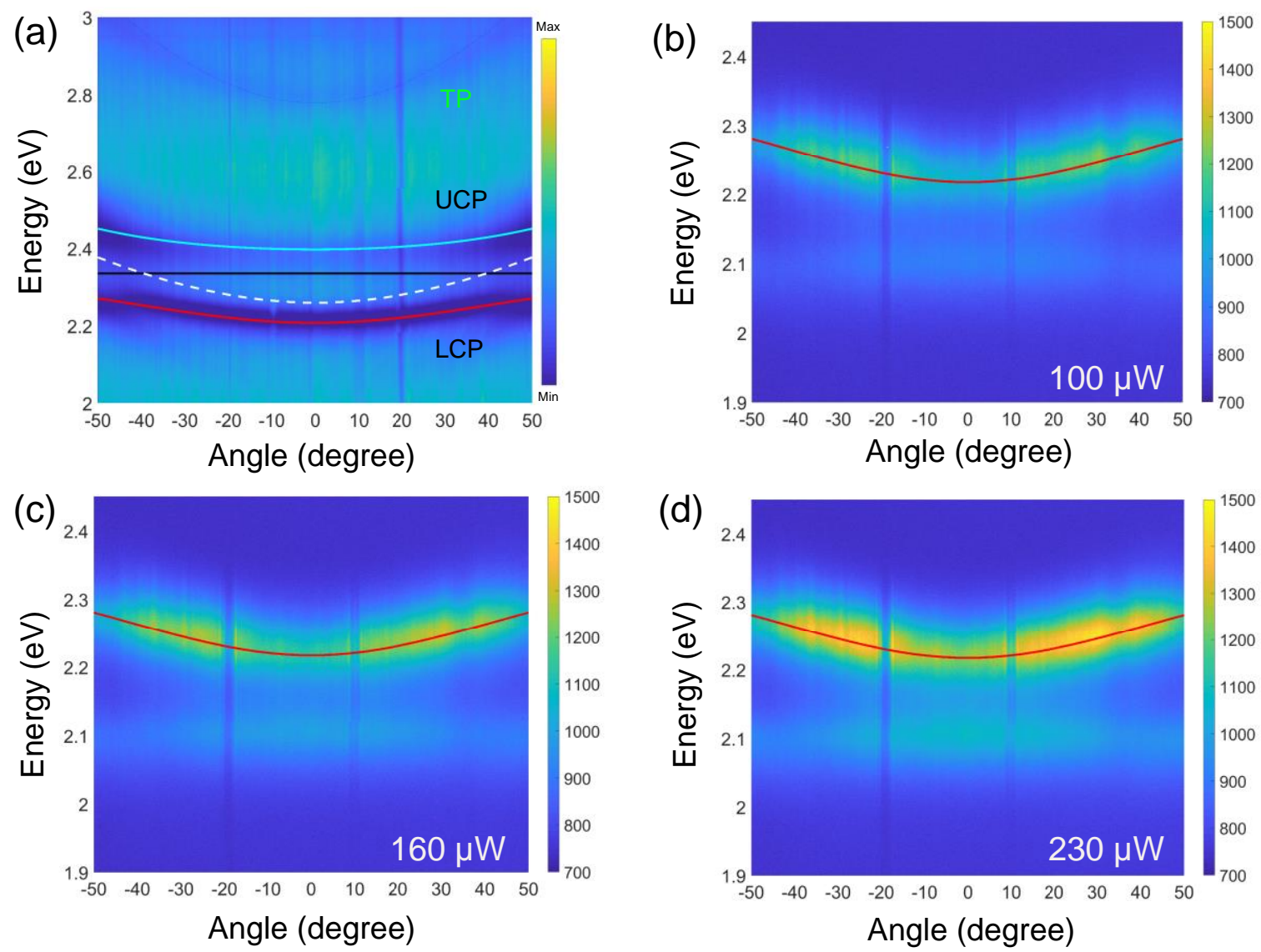

Figure S6. a) TM-polarized angle-resolved reflectivity map measured for the hybrid cavity with no top $\mathrm{Si}_{3} \mathrm{~N}_{4}$ layer (HC1). The solid black, dashed white, and dotted blue curve show the uncoupled exciton, cavity photon, and Tamm polariton (TP) dispersion, respectively. The solid cyan and red curve show the cavity polariton dispersion. Angle-resolved photoluminescence map: b) $100 \mu \mathrm{W}$, c) $160 \mu \mathrm{W}$, d) $230 \mu \mathrm{W}$. 

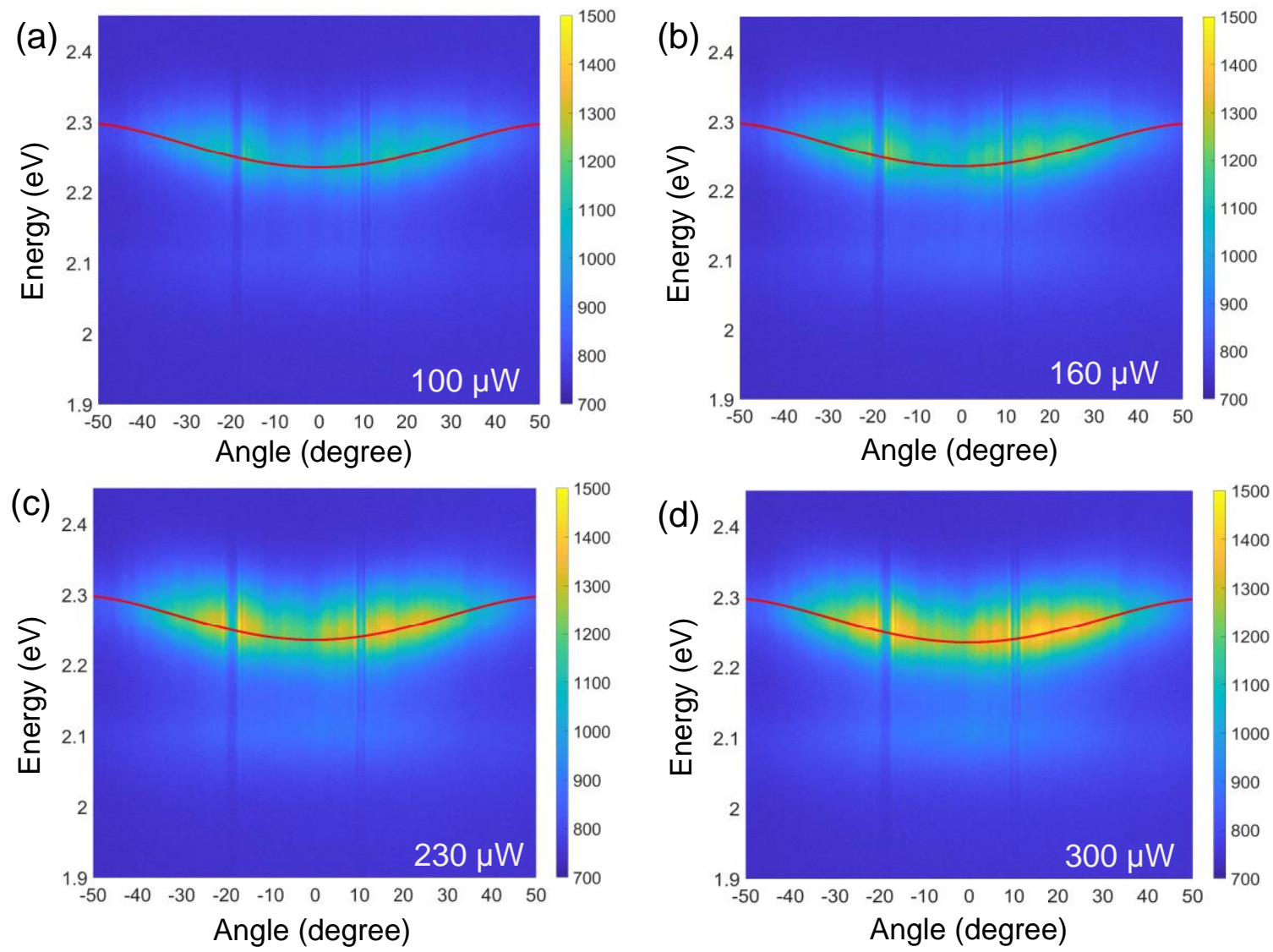

Figure S7. TE-polarized angle-resolved photoluminescence map measured for the hybrid cavity with a $30 \mathrm{~nm}$ top $\mathrm{Si}_{3} \mathrm{~N}_{4}$ layer (HC2) under different pump power: a) $100 \mu \mathrm{W}$, b) $160 \mu \mathrm{W}$, c) 230 $\mu \mathrm{W}$, d) $300 \mu \mathrm{W}$. 

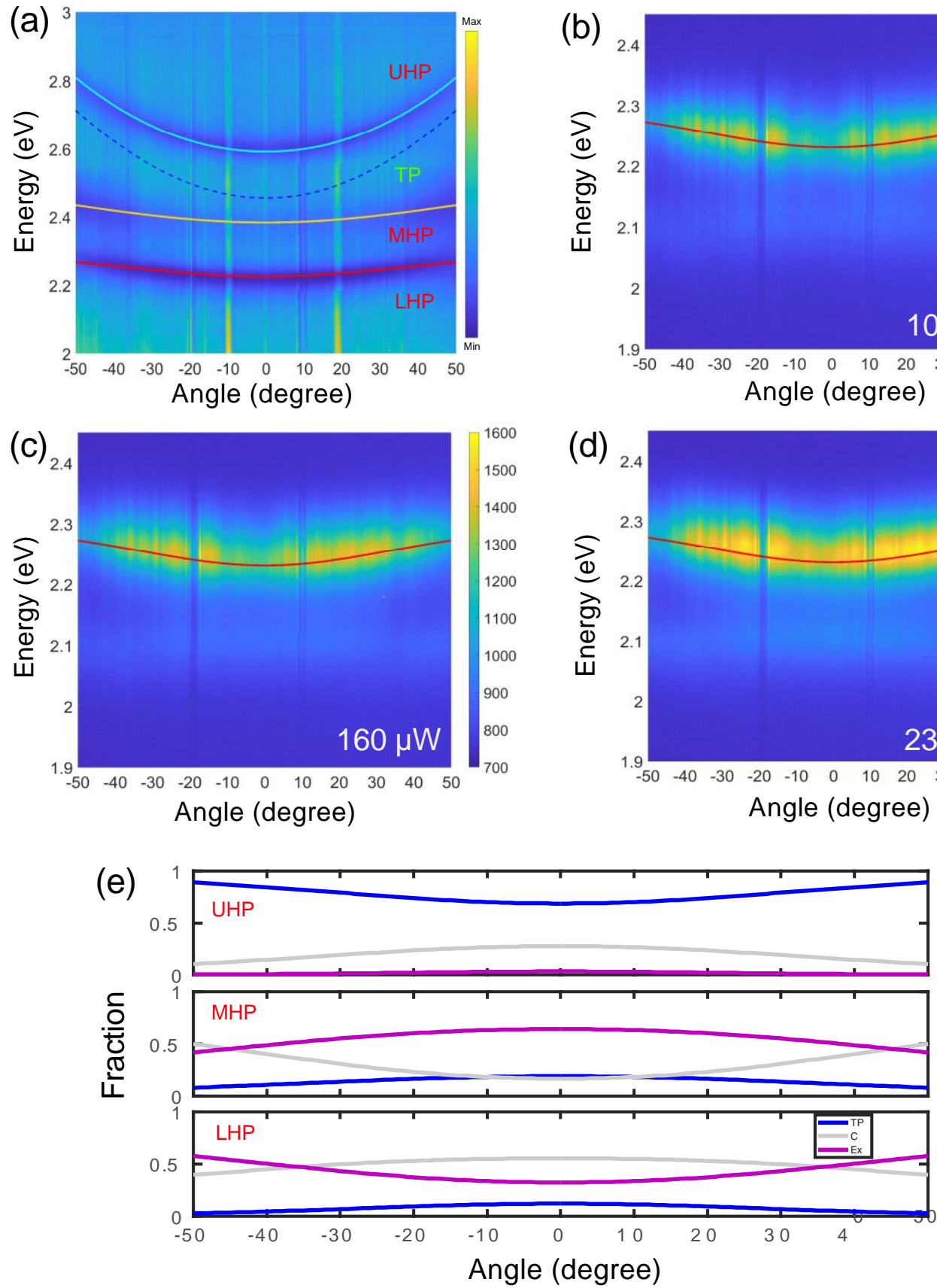
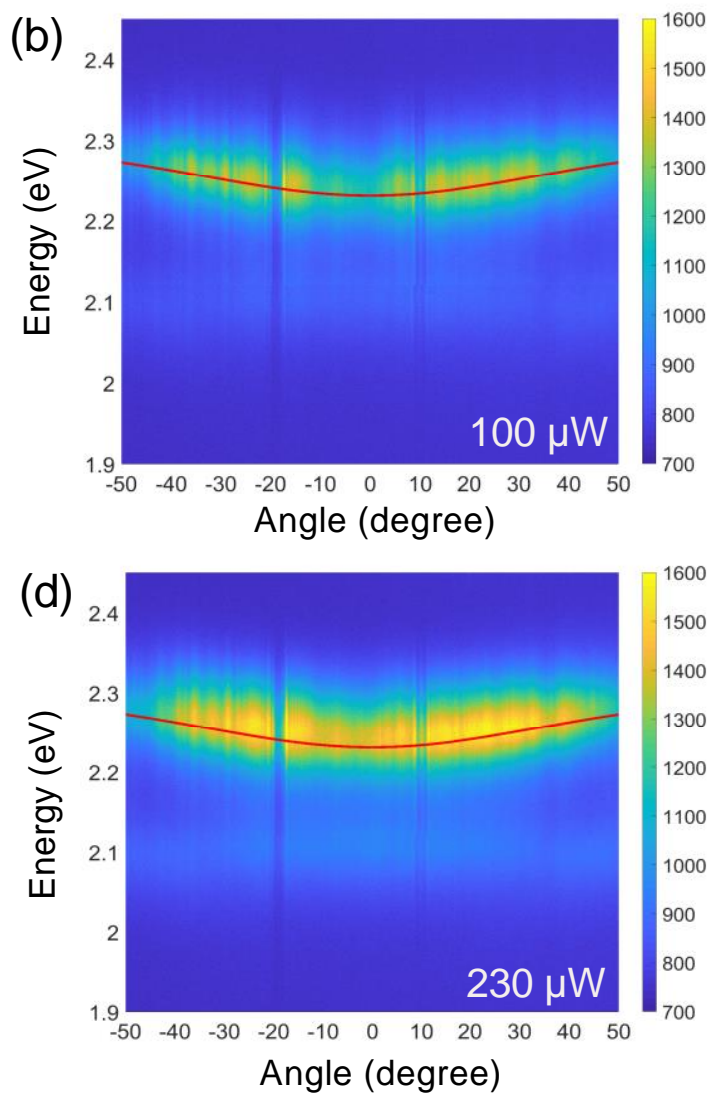

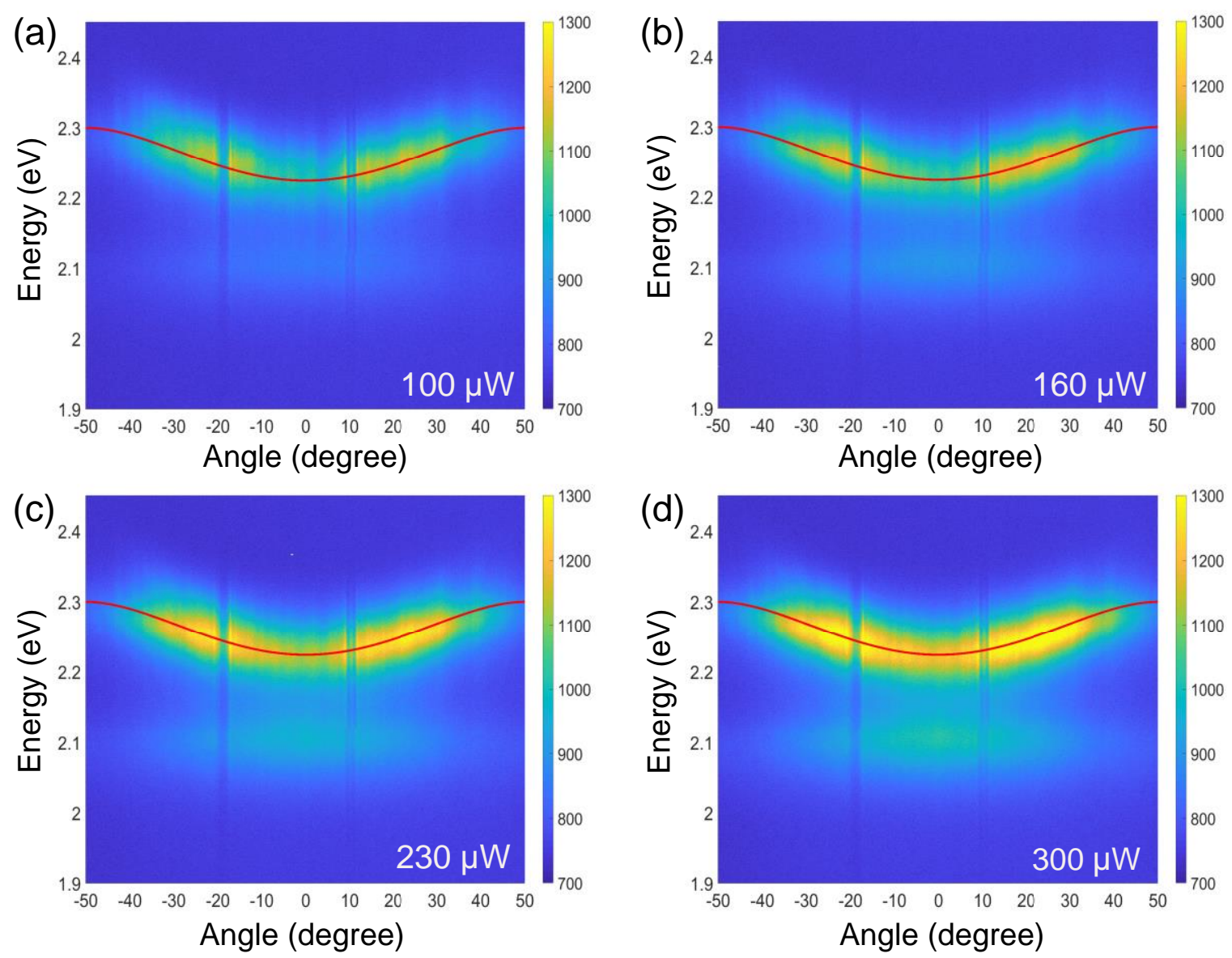

Figure S9. TE-polarized angle-resolved photoluminescence map measured for the hybrid cavity with a $50 \mathrm{~nm}$ top $\mathrm{Si}_{3} \mathrm{~N}_{4}$ layer (HC3) under different pump power: a) $100 \mu \mathrm{W}$, b) $160 \mu \mathrm{W}$, c) 230 $\mu \mathrm{W}$, d) $300 \mu \mathrm{W}$. 

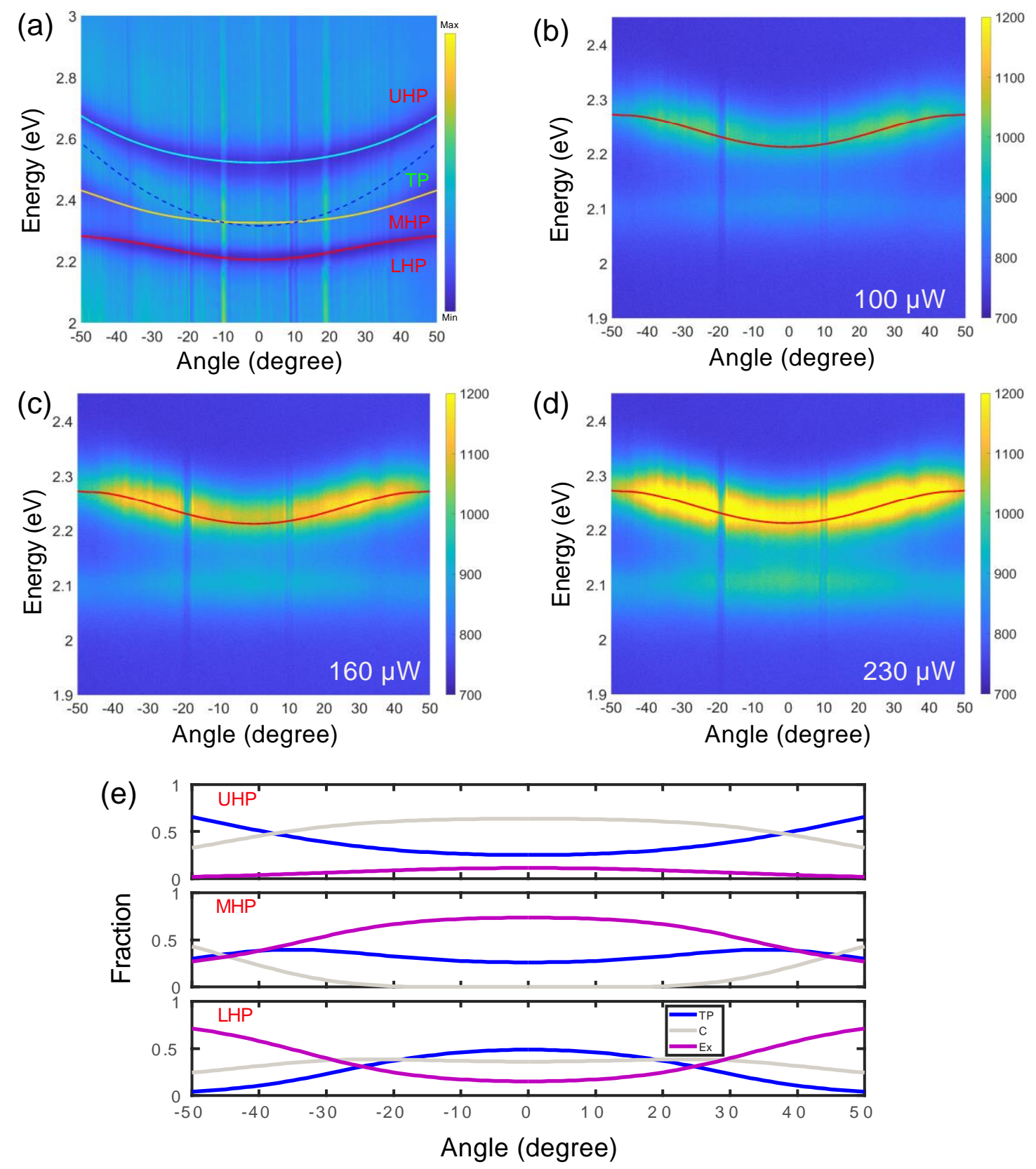

Figure S10. a) TM-polarized angle-resolved reflectivity map measured for the hybrid cavity with a $50 \mathrm{~nm}$ top $\mathrm{Si}_{3} \mathrm{~N}_{4}$ layer (HC3). The dashed blue curve show the uncoupled Tamm polariton (TP) dispersion. The solid cyan, orange, and red curve show the hybrid polariton dispersion. Angleresolved photoluminescence map: b) $100 \mu \mathrm{W}$, c) $160 \mu \mathrm{W}$, d) $230 \mu \mathrm{W}$. e) Hopfield coefficients $|\alpha|^{2}$, $|\beta|^{2}$, and $|\gamma|^{2}$ showing the composition of UHP, MHP, and LHP calculated using the coupled threeoscillator model. 
(a)

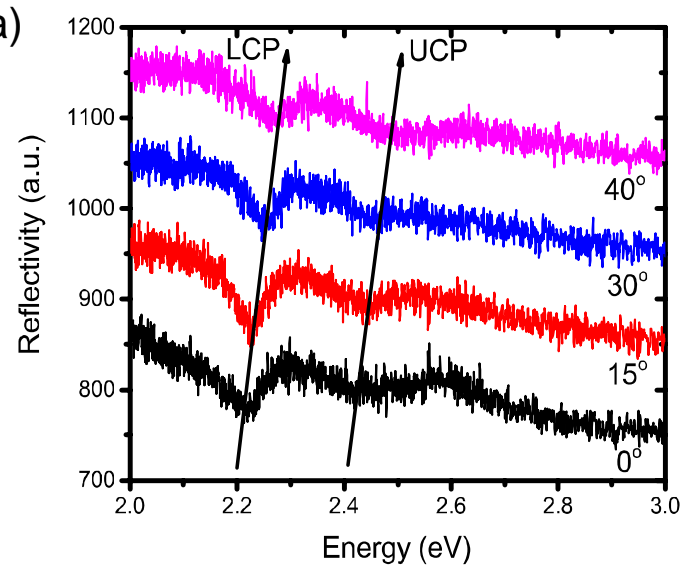

(c)

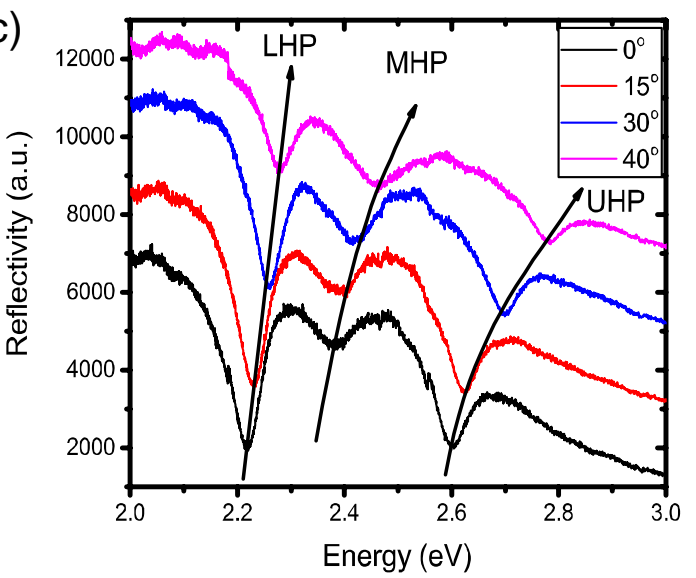

(b)

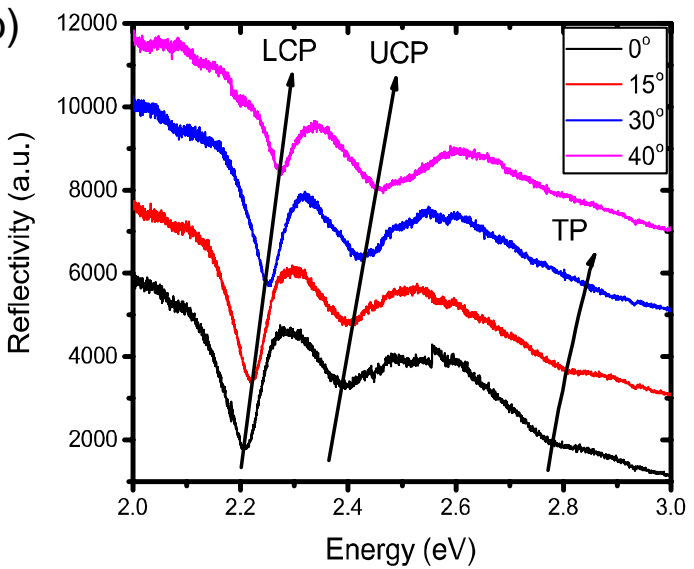

(d)

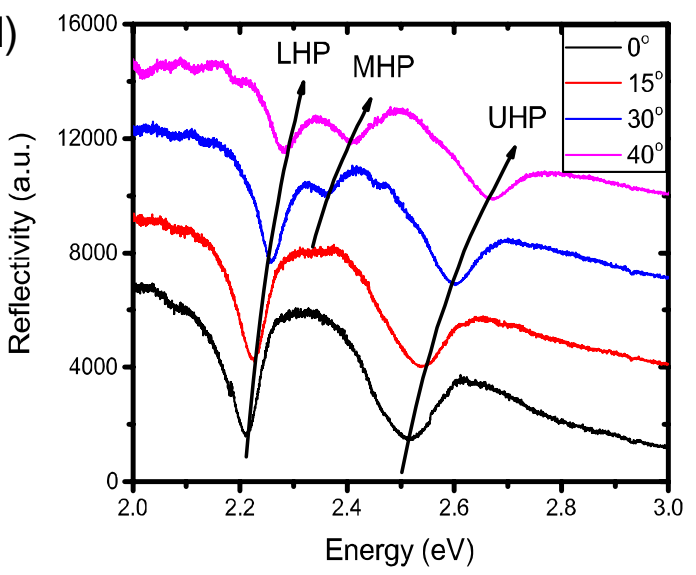

Figure S11. TE-polarized reflectivity spectra measured at several angles: a) metal cavity, b) HC1, c) $\mathrm{HC} 2$, d) $\mathrm{HC} 3$. 

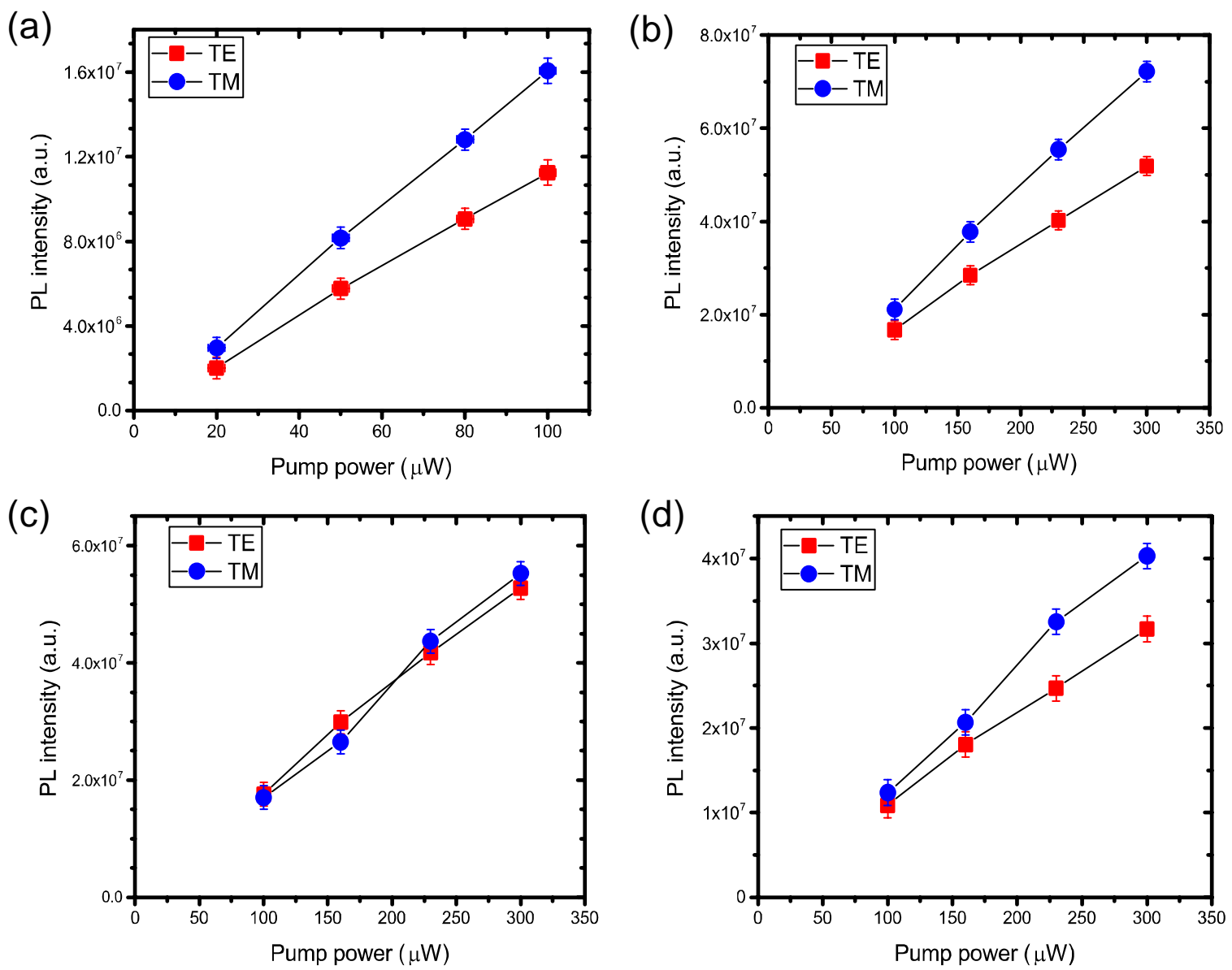

Figure S12. Power-dependent integrated PL intensity: a) metal cavity, b) HC1, c) HC2, d) HC3. 

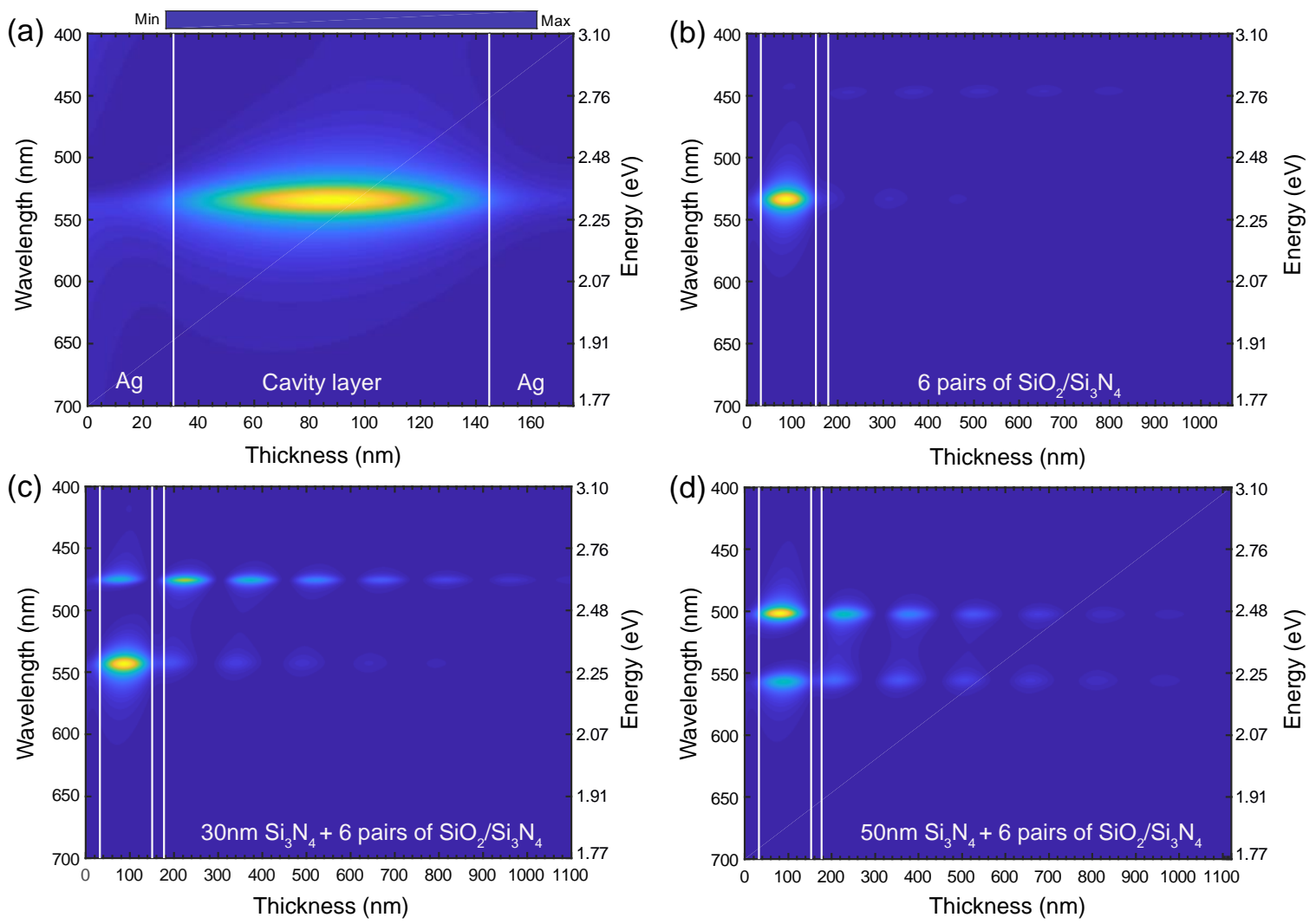

Figure S13. Simulated profiles of the electric-field-intensity distribution for cavities filled with $115 \mathrm{~nm}$ PS layer. a) Metal cavity, b) $\mathrm{HC} 1$ with no top $\mathrm{Si}_{3} \mathrm{~N}_{4}$ layer, c) $\mathrm{HC} 2$ with a $30 \mathrm{~nm}$ top $\mathrm{Si}_{3} \mathrm{~N}_{4}$ layer, d) $\mathrm{HC} 3$ with a $50 \mathrm{~nm}$ top $\mathrm{Si}_{3} \mathrm{~N}_{4}$ layer. 

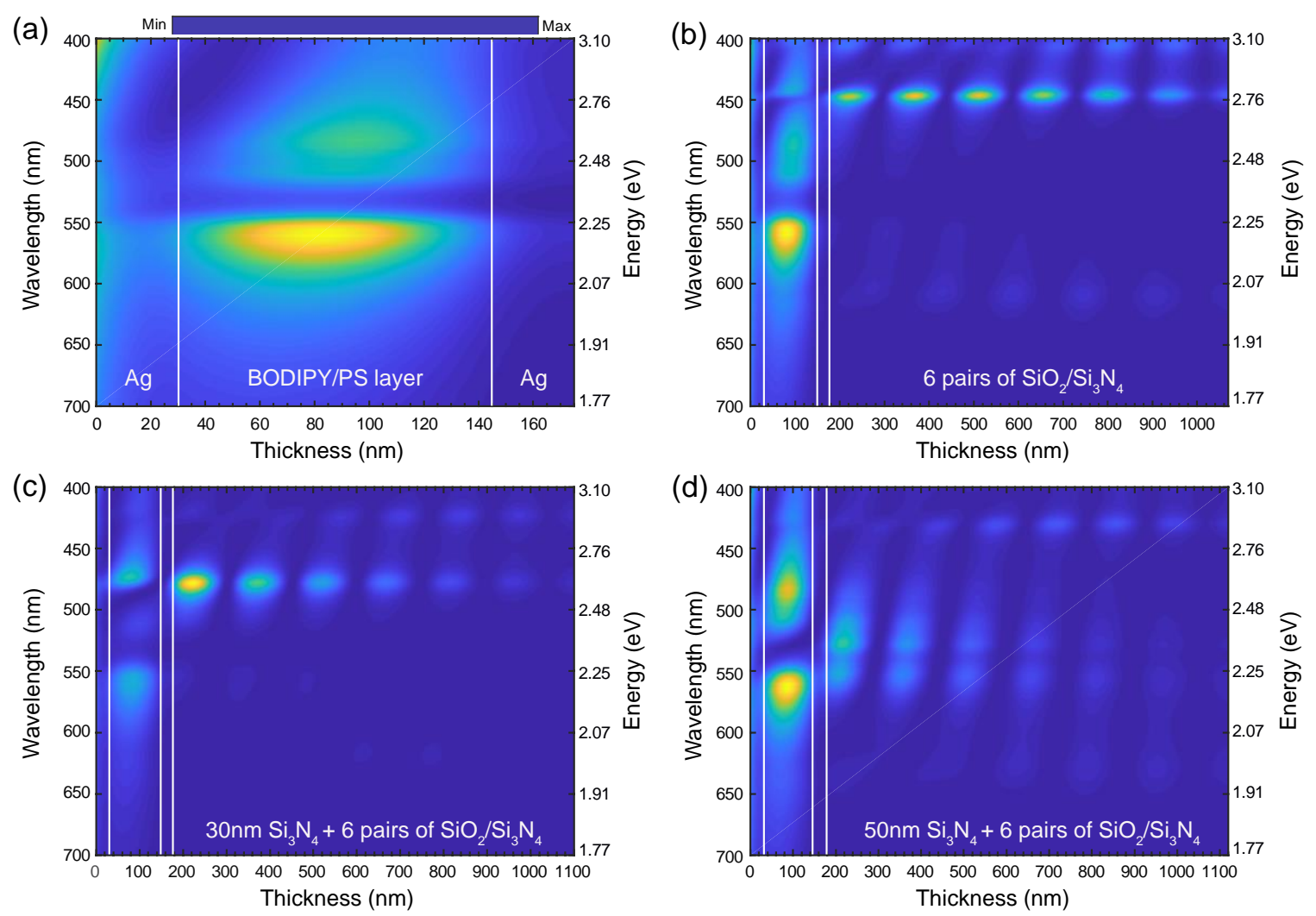

Figure S14. Simulated profiles of the electric-field-intensity distribution for cavities filled with $115 \mathrm{~nm}$ BODIPY/PS layer. a) Metal cavity, b) $\mathrm{HC} 1$ with no top $\mathrm{Si}_{3} \mathrm{~N}_{4}$ layer, c) $\mathrm{HC} 2$ with a $30 \mathrm{~nm}$ top $\mathrm{Si}_{3} \mathrm{~N}_{4}$ layer, d) $\mathrm{HC} 3$ with a $50 \mathrm{~nm}$ top $\mathrm{Si}_{3} \mathrm{~N}_{4}$ layer. 


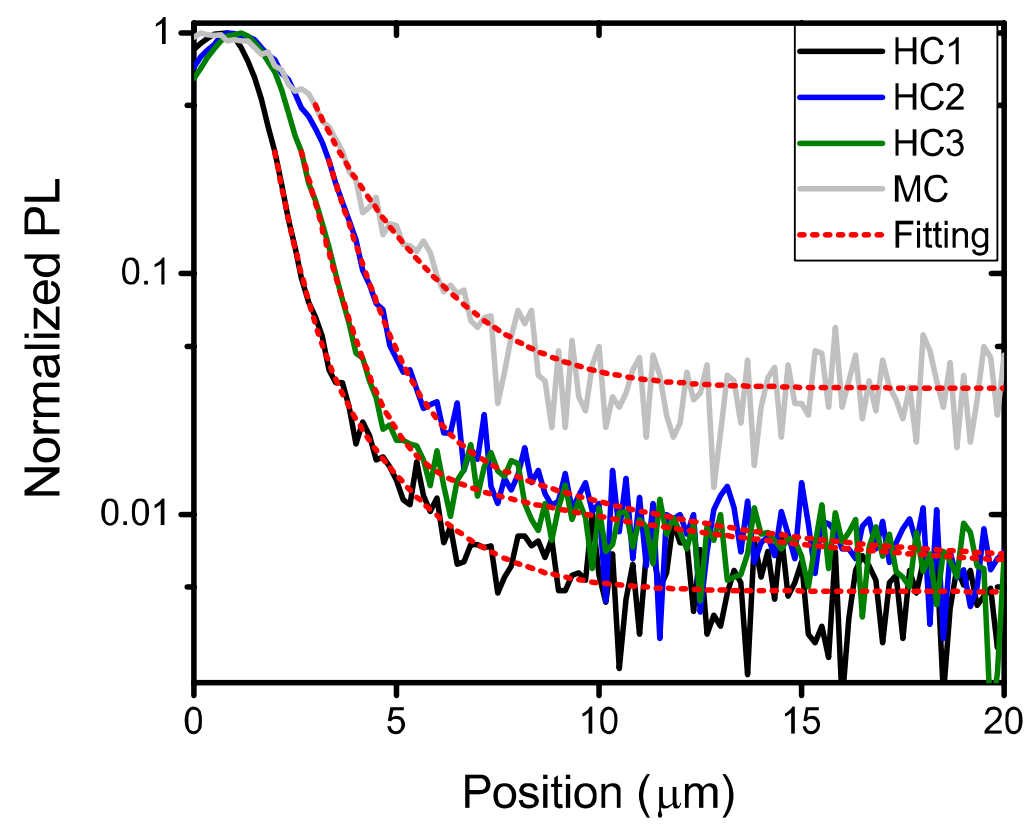

Figure S15. PL Intensity profiles (logarithmic scale) of polariton propagation at the wavelength of $555 \mathrm{~nm}$ from the bottom of lower polaritons for different cavities. 


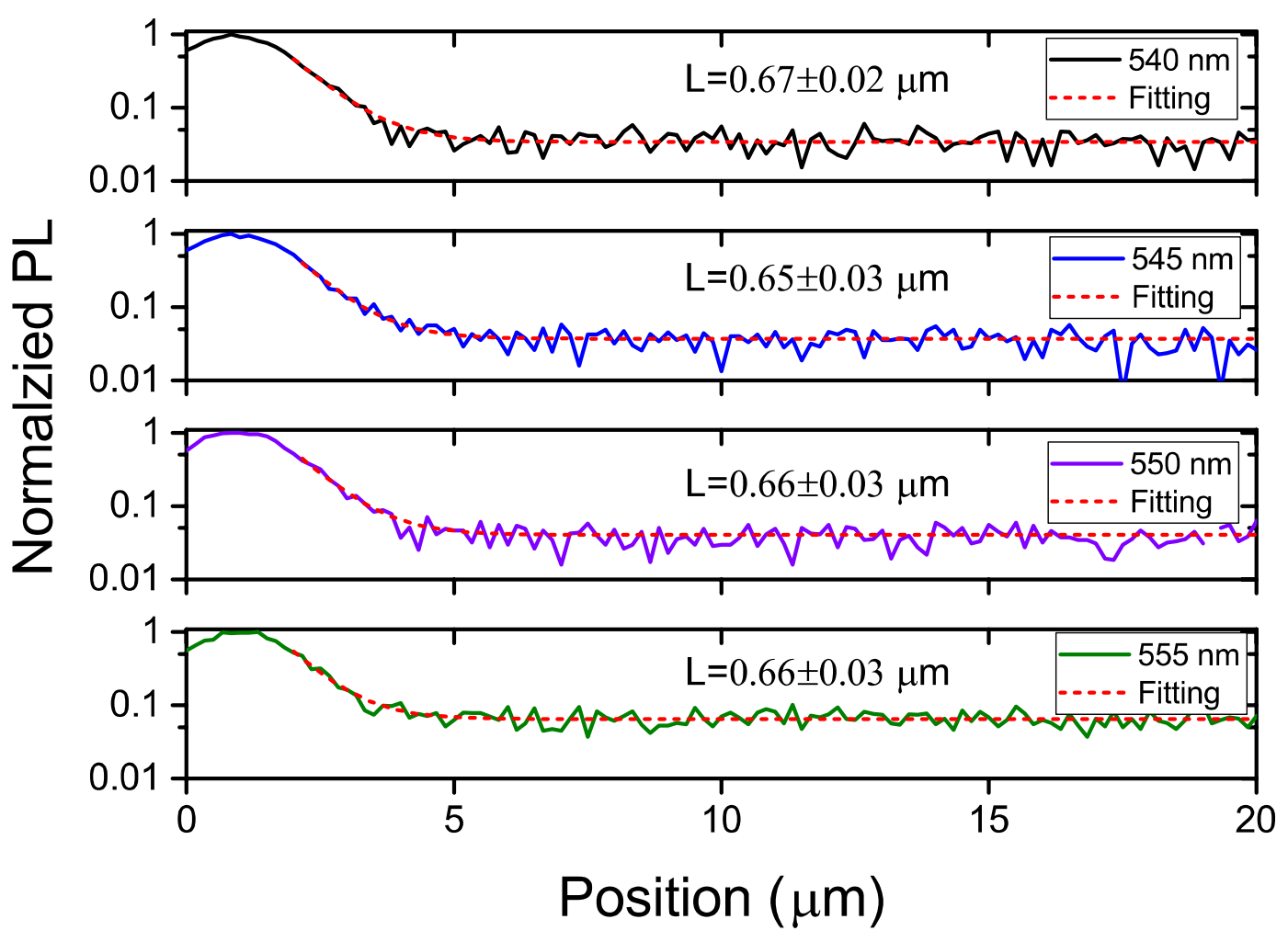

Figure S16. PL Intensity profiles of polariton propagation fit using one-exponential at different wavelengths from a $115 \mathrm{~nm}$ BODIPY/PS neat film. 


\begin{tabular}{ccccccccc}
\hline \multirow{2}{*}{ Cavity } & \multicolumn{2}{c}{$E_{0}(\mathrm{eV})$} & \multicolumn{2}{c}{$n_{\text {eff }}$} & \multicolumn{2}{c}{$V_{1}(\mathrm{meV})$} & \multicolumn{2}{c}{$V_{2}(\mathrm{meV})$} \\
\cline { 2 - 9 } Structure & $\mathrm{TE}$ & $\mathrm{TM}$ & $\mathrm{TE}$ & $\mathrm{TM}$ & $\mathrm{TE}$ & $\mathrm{TM}$ & $\mathrm{TE}$ & $\mathrm{TM}$ \\
\hline \multirow{2}{*}{$\mathrm{MC}$} & 2.27 & 2.28 & 1.66 & 2.88 & & & \multirow{2}{*}{$98.9 \pm 10$} & $97.4 \pm 10$ \\
& \pm 0.06 & \pm 0.06 & \pm 0.05 & \pm 0.05 & & & & \\
$\mathrm{HC} 1$ & 2.27 & 2.28 & 1.66 & 2.88 & & & $98.9 \pm 10$ & $97.4 \pm 10$ \\
& \pm 0.06 & \pm 0.06 & \pm 0.05 & \pm 0.05 & & & & \\
$\mathrm{HC} 2$ & 2.34 & 2.35 & 1.76 & 3.63 & & & & \\
& \pm 0.06 & \pm 0.06 & \pm 0.05 & \pm 0.05 & & & & \\
$\mathrm{HC3}$ & 2.40 & 2.41 & 1.74 & 3.31 & $127 \pm 15$ & $132 \pm 15$ & $87.6 \pm 10$ & $87.4 \pm 10$ \\
& \pm 0.06 & \pm 0.06 & \pm 0.05 & \pm 0.05 & & & & \\
& & & & & & & & \\
\end{tabular}

Table S1. Parameters for the coupled oscillator model used to fit polariton dispersion of metal cavity (MC), hybrid cavity $\mathrm{HC} 1, \mathrm{HC} 2$, and $\mathrm{HC} 3$. The uncoupled exciton energy of dye molecules is fixed at $2.33 \mathrm{eV}$ for all the cavities. For MC, a coupled two-oscillator model is used. For HC1, as Tamm polaritons are far off-resonant with cavity polaritons, there is no strong coupling, thus, the cavity polariton dispersion is also fit using a coupled two-oscillator model. For HC2 and HC3, a coupled three-oscillator model is used. It is worth mentioning that the substantial difference of the effective refractive index, $n_{\text {eff, }}$ between TE and TM polarization is due to the large polarization splitting of metal cavity. ${ }^{3}$ 


\begin{tabular}{ccccc}
\multicolumn{1}{c}{$\begin{array}{c}540 \\
\text { Sample }\end{array}$} & $\begin{array}{c}545 \\
(\mathrm{~nm})\end{array}$ & $\begin{array}{c}550 \\
(\mathrm{~nm})\end{array}$ & $\begin{array}{c}555 \\
(\mathrm{~nm})\end{array}$ \\
\hline Film & $\mathrm{L}=0.67 \pm 0.02$ & $\mathrm{~L}=0.65 \pm 0.03$ & $\mathrm{~L}=0.66 \pm 0.03$ & $\mathrm{~L}=0.66 \pm 0.03$ \\
MC & $\mathrm{L}=1.63 \pm 0.03^{*}$ & $\mathrm{~L}_{1}=0.68 \pm 0.02$ & $\mathrm{~L}_{1}=0.62 \pm 0.01$ & $\mathrm{~L}_{1}=0.49 \pm 0.02$ \\
& & $\mathrm{~L}_{2}=1.74 \pm 0.10$ & $\mathrm{~L}_{2}=1.55 \pm 0.10$ & $\mathrm{~L}_{2}=1.71 \pm 0.05$ \\
HC1 & $\mathrm{L}_{1}=0.35 \pm 0.05$ & $\mathrm{~L}_{1}=0.41 \pm 0.02$ & $\mathrm{~L}_{1}=0.42 \pm 0.02$ & $\mathrm{~L}_{1}=0.42 \pm 0.01$ \\
& $\mathrm{~L}_{2}=0.91 \pm 0.20$ & $\mathrm{~L}_{2}=1.17 \pm 0.13$ & $\mathrm{~L}_{2}=1.51 \pm 0.12$ & $\mathrm{~L}_{2}=1.59 \pm 0.11$ \\
HC2 & $\mathrm{L}_{1}=0.68 \pm 0.06$ & $\mathrm{~L}_{1}=0.80 \pm 0.03$ & $\mathrm{~L}_{1}=0.75 \pm 0.02$ & $\mathrm{~L}_{1}=0.74 \pm 0.02$ \\
& $\mathrm{~L}_{2}=2.02 \pm 0.44$ & $\mathrm{~L}_{2}=4.20 \pm 0.20$ & $\mathrm{~L}_{2}=4.33 \pm 0.30$ & $\mathrm{~L}_{2}=4.83 \pm 0.32$ \\
HC3 & $\mathrm{L}_{1}=0.58 \pm 0.03$ & $\mathrm{~L}_{1}=0.66 \pm 0.01$ & $\mathrm{~L}_{1}=0.63 \pm 0.01$ & $\mathrm{~L}_{1}=0.64 \pm 0.01$ \\
& $\mathrm{~L}_{2}=2.81 \pm 0.30$ & $\mathrm{~L}_{2}=4.66 \pm 0.15$ & $\mathrm{~L}_{2}=5.24 \pm 0.35$ & $\mathrm{~L}_{2}=6.35 \pm 0.30$ \\
\hline
\end{tabular}

Table S2. Fitting constants of the propagation length for neat film, metal cavity (MC), hybrid cavity $\mathrm{HC} 1, \mathrm{HC} 2$ and $\mathrm{HC} 3$. The data of neat film are fit using one-exponential function. For all the cavity samples, the data are fit using bi-exponential function. The unit of the propagation length is micron. *One-exponential is used as bi-exponential fitting gives two equal fitting constants. 
Reference:

(1) Chakraborty, B.; Gu, J.; Sun, Z.; Khatoniar, M.; Bushati, R.; Boehmke, A. L.; Koots, R.; Menon, V. M. Control of Strong Light-Matter Interaction in Monolayer WS2 through Electric Field Gating. Nano Lett. 2018, 18 (10), 6455-6460.

(2) Lerario, G.; Ballarini, D.; Fieramosca, A.; Cannavale, A.; Genco, A.; Mangione, F.; Gambino, S.; Dominici, L.; De Giorgi, M.; Gigli, G.; Sanvitto, D. High-Speed Flow of Interacting Organic Polaritons. Light Sci. Appl. 2017, 6 (2), e16212-6.

(3) Kéna-cohen, S.; Maier, S. A.; Donal, D. C. Ultrastrongly Coupled Exciton - Polaritons in Metal-Clad Organic Semiconductor Microcavities. 2013, 1, 827-833. 